\title{
The Research Of Fluid-structure Interaction Of The Hydrocyclone Under The Condition Of Vibration
}

\author{
XU Dekui \\ Oil Production Engineering Research Institute of Daqing Oilfield Company Limited, Daqing 163453, China
}

\begin{abstract}
During the operation of the hydrocyclone, vibrations are often generated by internal fluids and external disturbances resulting in fluid-structure interaction, causing the spatiotemporal evolution of the flow field and the movement of the structure. In this paper, the flow characteristic and the structural motion of the periodic vibrating hydrocyclones are studied. The bidirectional fluid-solid model of hydrocyclone under vibration condition is established. The flow field and structure motion under different vibration frequencies and structure resonances are studied. It shows that the velocities in the three directions oscillate positively and negatively with the motion of structure, the amplitude of the oscillation is the largest on resonance, the skewing of the velocity in the flow field is smaller than the structure; the tangential velocity is asymmetric and the radial velocity is increased significantly, the deformation of the structure is different on the different vibration frequencies, which causes the flow field of distribution of each section to be different. This study will provide the theoretical guidance for the application of hydrocyclone under the vibration conditions.
\end{abstract}

\section{Introduction}

The hydrocyclone is widely used as a separation equipment in the field of petroleum, petrochemical and sewage treatment. During the operation, it is caused vibration by internal fluid or external disturbance. The fluid-structure interaction under vibration conditions will cause the spatiotemporal evolution of the flow field and the movement of the structure, affecting the separation efficiency and the structural strength of equipment.

Pei $\mathrm{Ji}^{[1,2]}$ used two-way synchronous solution method to study the influence of the impeller fluid-solid coupling on the internal flow field of the centrifugal pump. Xu Feng ${ }^{[3]}$ used ANSYS Workbench platform to obtain the flow field and velocity distribution in the pump cavity by fluid-solid coupling simulation. Satish Kumar Chimakurthi $^{[4]}$ used ANSYS Workbench platform to study the fluid structure interactions and validate them. Zhu $\mathrm{Li}^{[5]}$ used the standard $\mathrm{k}-\varepsilon$ turbulence model and fluid-solid coupling technique to calculate the stress distribution of the axial pump impeller. $\mathrm{Li} \mathrm{Wei}^{[6]}$ analyzed the mechanical properties of the mixed-flow pump impeller by bidirectional fluid-solid coupling, and the fluid adopts the standard k- $\varepsilon$ turbulence model, the structural response uses the elastic structure dynamics equation. Liang Quanwei ${ }^{[7]}$ used the fluid-solid coupling method to analyze the modality of the runner in the water medium, and obtained the resonance effect of the fluid-solid coupling on the rotor. Onur Celikkan ${ }^{[8]}$ used the fluid-solid coupling technique to analyze the coupling behavior of the freezer and the duct, and studied the vibration between the structure and the cavity in the freezer to avoid resonance. Qiu Yadong ${ }^{[9]}$ used the two-way coupling implicit algorithm to study the vibrations characteristics of hydrocyclones on support or not. Sen $\mathrm{Li}^{[10]}$ conducted a numerical simulation study on the coupled flow field of a hydrocyclone under self-excited vibration conditions, and compared the flow field distribution, indicating that the influence of coupling on the flow field in the cyclone can not be ignored. $\mathrm{Xu}$ $\mathrm{Yan}^{[11,12]}$ studied the fluid-solid of the spiral flow in the cyclone under the vibration condition with the same excitation frequency. It is concluded that the sporal flow field moves along with the structure and the motion of the structure rises the skewing of the flow field structure.

At present, domestic and foreign scholars have carried out relevant researches on fluid machinery based on the fluid-solid coupling theory. There are few researches on the relationship between the structural motion and flow field of periodic vibrating cyclone separator, especially the influence of resonance on it. So it is necessary to study the fluid-solid coupling effect of cyclone separator under different vibration frequencies and resonance conditions.

\section{Computational modeling and algorithms}

\subsection{Flow field computing model and algorithms}

\subsubsection{Fluid domain control equation}

${ }^{*}$ Corresponding author: xudekui@petrochina.com.cn 
Mass conservation equation:

$$
\frac{\partial \rho}{\partial t}+\frac{\partial}{\partial x_{j}}\left(\rho u_{j}\right)=0
$$

Formula: $\rho$ Fluid density, $u_{j}$ the component of velocity in the coordinate direction, where $j=1,2,3$.

Momentum conservation equation:

$$
\frac{\partial u_{i}}{\partial t}+\frac{\partial}{\partial x_{j}}\left(\rho u_{i} u_{j}\right)=-\frac{\partial p}{\partial x_{i}}+\frac{\partial}{\partial x_{j}}\left(\mu \frac{\partial u_{i}}{\partial x_{j}}-\rho \overline{u_{i}^{\prime} u_{j}^{\prime}}\right)
$$

Formula: $\mu$ for fluid kinematic viscosity, $\overline{u_{i}^{\prime} u_{j}^{\prime}}$ for Reynolds stress.

Reynolds stress model (RSM) is used to close the governing equation.

\subsubsection{Geometric model and algorithms}

In the fluid domain, a double-cone hydrocyclone with main diameter of $\mathrm{D}=28 \mathrm{~mm}$ is selected, and its structure diagram is shown in Fig. 1. The inlet depth, width and length are $\mathrm{a}=0.68 \mathrm{D}, \mathrm{b}=0.7 \mathrm{D}, \mathrm{c}=2.56 \mathrm{D}$, the overflow nozzle diameter $\mathrm{Du}=0.28 \mathrm{D}$, the underflow diameter $\mathrm{Dd}=0.5 \mathrm{D}$, the length of the swirl chamber $\mathrm{h} 1=\mathrm{D}$, the length of the large cone segment $\mathrm{h} 2=1.42 \mathrm{D}$, the length of the small cone segment $h 3=9.55 \mathrm{D}$, and the length of the tail tube $\mathrm{h} 4=10.18 \mathrm{D}$, respectively. Big cone angle $\mathrm{a}=20$ degrees, small cone angle theta $=1.5$ degrees. The fluid medium is water, the inlet is velocity inlet, the bottom flow and overflow are free outflow, the split ratio is $5 \%$, the uncoupled case is standard wall function, and the coupling case is the coupling interface. The governing equation is discretized by finite volume method, the pressure and

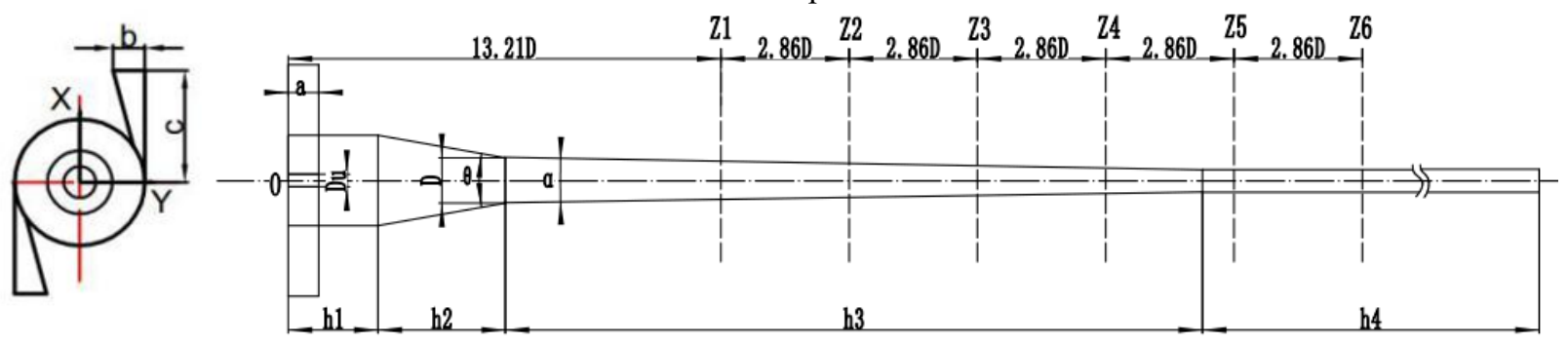

Fig. 1. Schematic diagram for hydrocyclone geometry

velocity are coupled by SIMPLEC algorithm, and the pressure term is discretized by PRESTO! The momentum equation is discretized by QUICK scheme with third-order accuracy, turbulent kinetic energy and dissipation rate by second-order upwind scheme, and Reynolds stress by first-order upwind scheme.

\subsubsection{Mesh generation and independence computing}

ICEM16.0 is used for meshing. The mesh of fluid field is shown in Figure 2. In order to reduce computational time under the condition of computational accuracy, the grid independence analysis is carried out according to the steady flow field.

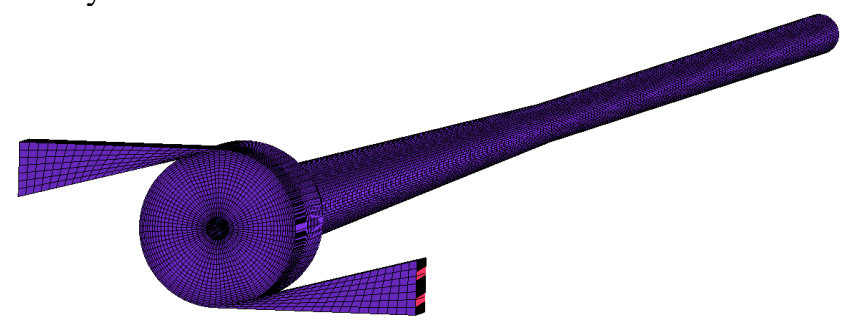

Fig. 2. Meshed hydrocyclone geometry in fluid domain

Four kinds of grids are solved in steady state, and their convergence rates are compared. The tangential velocity at the section of 3.84D is shown in Fig. 3.

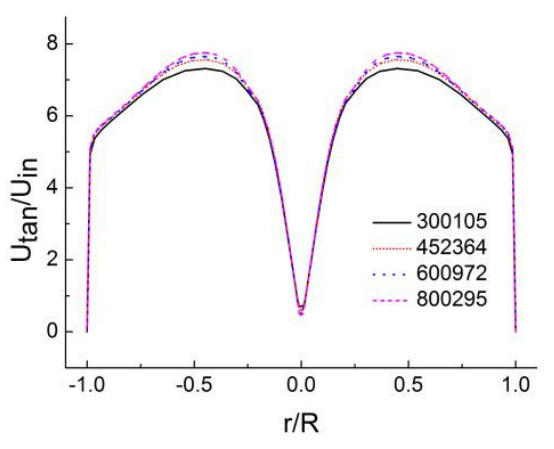

Fig. 3. Tangential velocities of different grid at $3.84 \mathrm{D}$ sections

As can be seen from Figure 3, the maximum tangential velocity increases with the increase of the number of grids. When the number of grids is 600972 , the error is $1.2 \%$ when the number of grids is 800295 . Therefore, the number of grids can be 600927 .

\subsection{Solid domain computing method}

The finite element method is used to discretize the structure. The structure vibrates under the combined action of internal fluid and external exciting force. The motion equation of the hydrocyclone can be expressed as follows:

$$
[M]\{\ddot{\delta}\}+[C]\{\dot{\delta}\}+[K]\{\delta\}=\left\{F_{f}\right\}+\left\{F_{o}\right\}
$$

In the formula: $[\mathrm{M}]$ is the mass matrix; $[\mathrm{C}]$ is the damping matrix; $[\mathrm{K}]$ is the stiffness matrix; $\left\{F_{f}\right\}$ is the 
load of the fluid on the structure; $\left\{F_{o}\right\}$ is the load of the external changing with time; $\{\delta\},\{\dot{\delta}\},\{\ddot{\delta}\}$ are displacement vector,

velocity vector and acceleration vector varying with time respectively.

The structural element is solid186, and the two ends of the structure are constrained by fixed ends. The Newmark time integral is used to solve the transient dynamic equation.

\subsection{Fluid-solid coupling algorithms}

Ansys Workbench 16.0 is used to establish Fluent + Transient Structure fluid-structure coupling model. The numerical simulation of fluid-structure coupling is carried out by using the calculation method of staggered iterative coupling solution. The governing equations of fluid domain and solid domain satisfy the conditions of displacement coordination and force balance at the interface. The sinusoidal periodic excitation force is applied to the structure, and the pressure load on the wall is transferred to the structure by the fluid. The structure solves the deformation under the interaction of the excitation force and the fluid load according to the dynamic motion equation, and then transfers the displacement to the fluid. The ALE method is used to deal with the motion of the coupled interface boundary and the dynamic mesh. Smoothing, Layering and Remeshing methods are used to reconstruct the flow field, and a new boundary condition is adopted to calculate the flow field, and a new pressure distribution along the wall is obtained to realize the fluid-solid coupling calculation.

\section{Results analysis}

\subsection{Modal analysis of structures}

Table 1. The frequency of each order mode of fluid-solid coupling model

\begin{tabular}{c|c|c|c}
\hline Mode & 1 & 2 & 3 \\
\hline frequency $(\mathrm{Hz})$ & 33.36 & 87.37 & 164.54 \\
\hline
\end{tabular}

In order to select the appropriate vibration frequency, the modal analysis of the structure with Organic glass as the structural material and additional fluid mass is carried out, and the frequencies of each order of modal are shown in Table 1.

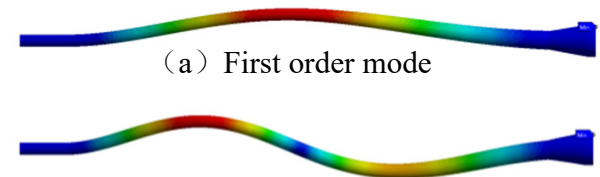

(b) Second-order modes

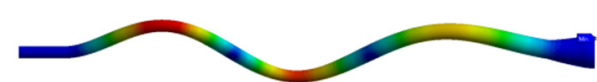

(c) Third-order modes
Fig. 4 shows the first three order vibration modes of the variable-diameter circular pipe structure of the hydrocyclone. It can be seen from the figure that the maximum deformation position of the structure is near the intersection of the small cone section and the tailpipe section in the first mode. In the second-order mode, the deformation of the structure is larger in the middle of the small cone section and the tail pipe section. In the third mode, the deformation mainly occurs at the small cone segment. Therefore, in the following study, the cross sections of the small cone section and the tailpipe section are mainly analyzed.

\subsection{Flow field velocity analysis}

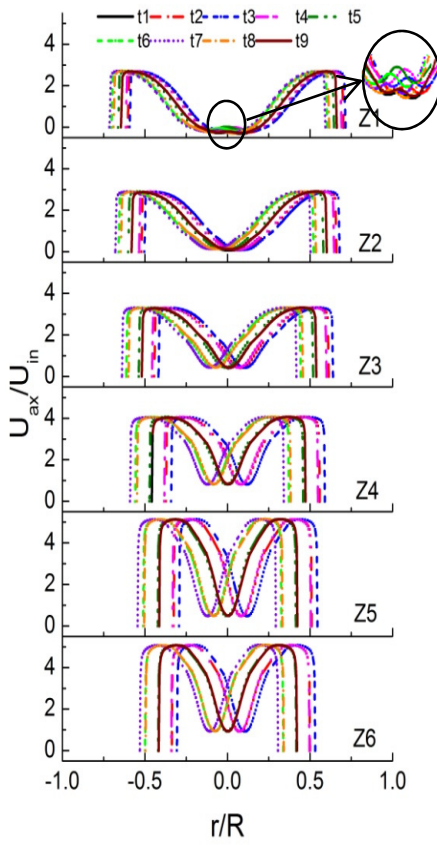

(a) $33.36 \mathrm{~Hz}$

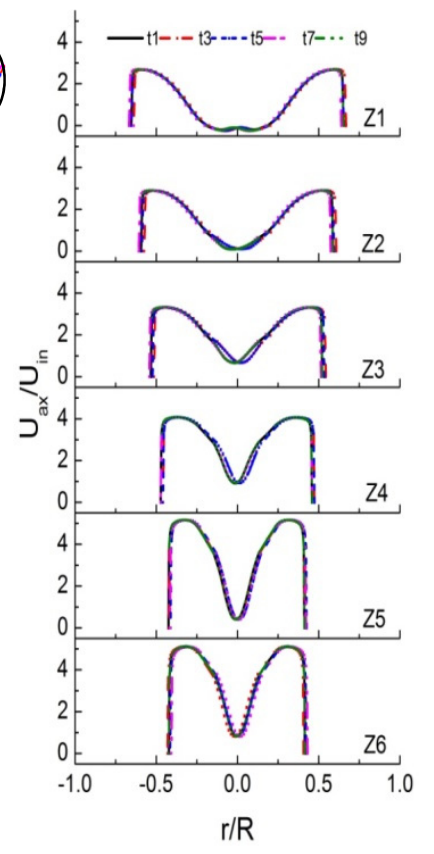

(b) $100 \mathrm{~Hz}$
Fig. 5. Axial velocity along $Y$ axis

The fluid-structure coupling parameters simulated in this chapter are set to the excitation force of $20 \mathrm{~N}$, and the excitation frequencies are $33.36 \mathrm{~Hz}, 100 \mathrm{~Hz}$ and $200 \mathrm{~Hz}$, respectively. The direction of excitation is $\mathrm{Y}$ axis, and the excitation position is $3.64 \mathrm{D}$. The flux is set at $3 \mathrm{~m}^{3} / \mathrm{h}$.

Axial velocities along the $\mathrm{Y}$-axis at different frequencies are shown in Fig. 5. At $33.36 \mathrm{~Hz}$, the structure deforms sharply due to resonance, which results in the largest displacement of axial velocity. At $100 \mathrm{~Hz}$, the structure is a second-order mode of vibration, and the displacement of axial velocity decreases significantly. The axial velocity in the core region shifts with the change of structural amplitude, and it is also affected by turbulence disturbance and asymmetry in the flow field. The displacement of the axial velocity in the flow field is slightly smaller than that in the displacement deformation of the structure.

Fig. 4. Modal modes of hydrocyclones 


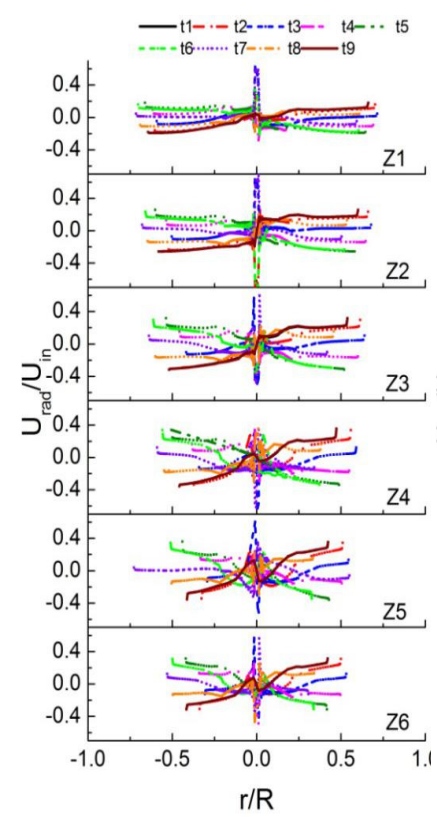

(a) $33.36 \mathrm{~Hz}$

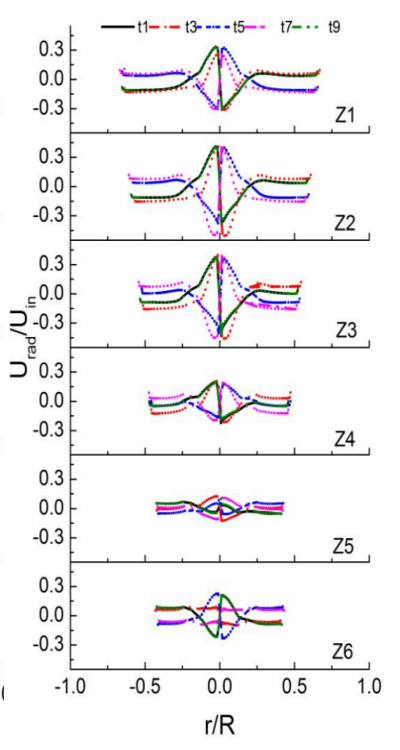

(b) $100 \mathrm{~Hz}$
Fig. 6. Radial velocity along $\mathrm{Y}$ axis

The radial velocity along the $\mathrm{Y}$ axis at different frequencies is shown in Fig. 6. Periodic excitation makes the radial velocity move from the traditional side wall to the center and change into positive and negative motion along the $\mathrm{Y}$ axis. At $33.36 \mathrm{~Hz}$, the radial velocity is the largest and the variation of the radial velocity in the axis region is disordered under the influence of structural resonance. At $100 \mathrm{~Hz}$, the radial velocity decreases obviously. Because of the second-order mode of the structure, the radial velocity of each section has a complex change.

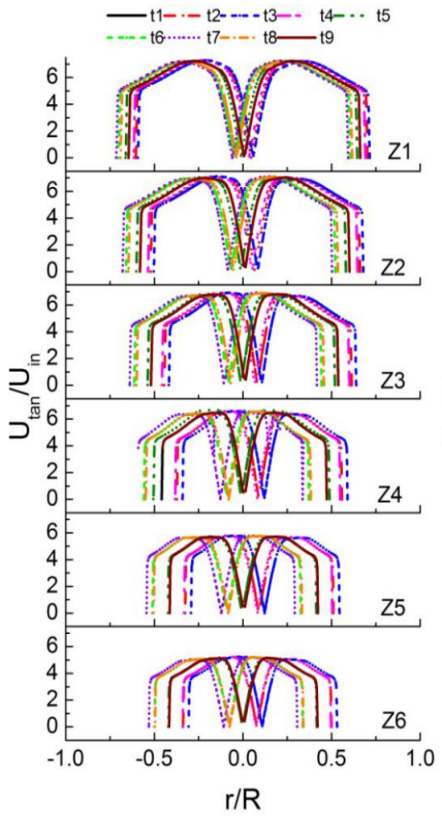

(a) $33.36 \mathrm{~Hz}$

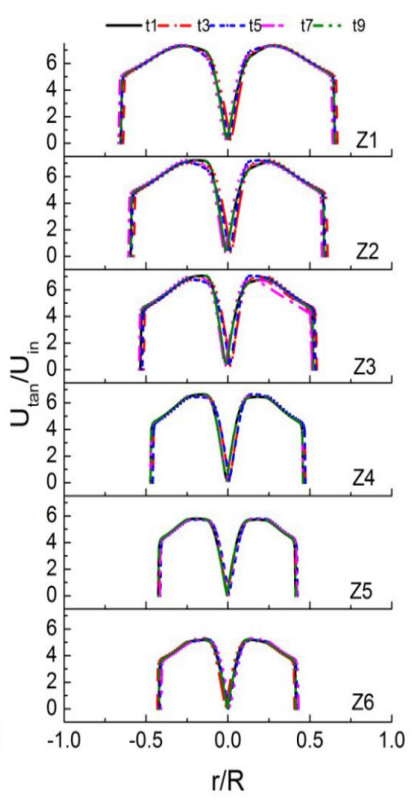

(b) $100 \mathrm{~Hz}$
Fig. 7. Tangential velocity along $\mathrm{Y}$ axis
The tangential velocity of $\mathrm{Y}$ axis at different frequencies is shown in Fig. 7. The periodic excitation force has little influence on the distribution and magnitude of tangential velocity in the flow field, but the offset of velocity axis occurs. At $33.36 \mathrm{~Hz}$, the tangential velocity offset is the largest, which is less than the structural vibration displacement due to the coupling effect of helical flow field. At $100 \mathrm{~Hz}$, the tangential velocity migration is small, and there is obvious asymmetry at different times. Because of the influence of the axial and radial velocity of the flow field, the tangential velocity of the axial region is deflected.

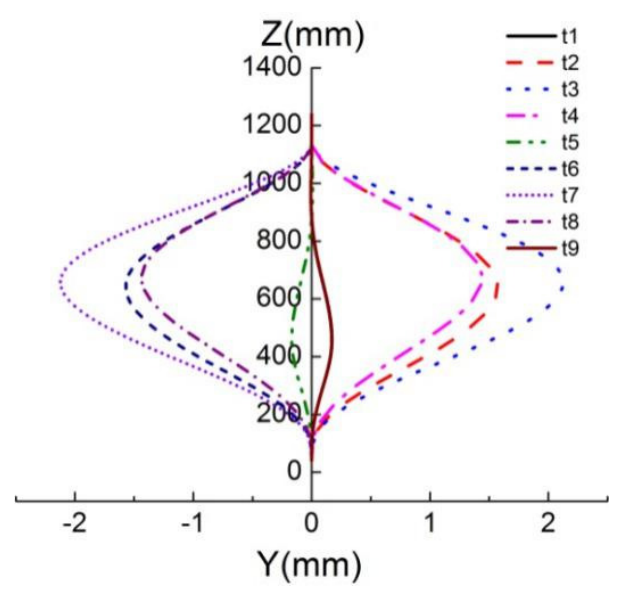

(a) $33 \cdot .36 \mathrm{~Hz}$

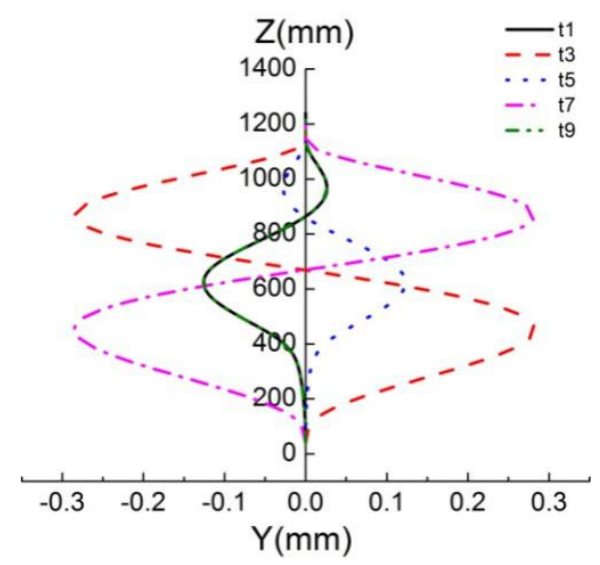

(b) $100 \mathrm{~Hz}$

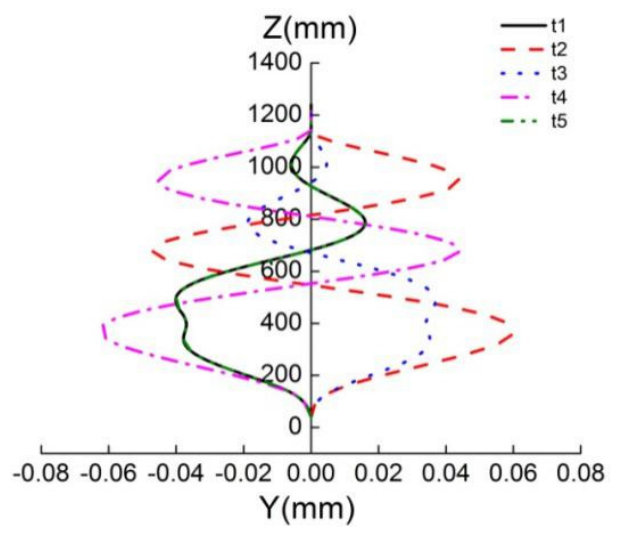

(c) $200 \mathrm{~Hz}$ 
Fig. 8. Shape diagram of $Y$-axis vibration

Figure 8 shows the vibration pattern of Y-axis. It can be seen from the figure that the vibration pattern of the hydrocyclone under the excitation force of $33.36 \mathrm{~Hz}$ is parabolic, and the vibration at different altitudes is basically synchronous. $100 \mathrm{~Hz}$ presents second-order mode and $200 \mathrm{~Hz}$ presents third-order mode.

\section{Conclusions}

1) The modal analysis of the hydrocyclone shows that the first three natural frequencies of the structure are 33.36 Hz, 87.37 Hz and 164.54 Hz.

2) The change of excitation frequency has little influence on the axial velocity distribution of the flow field. The maximum axial velocity values are basically the same, the velocity axis is offset, and the maximum axial velocity offset is $33.36 \mathrm{~Hz}$. The radial velocity is greatly affected by the structure motion, and the numerical value is related to the displacement of the structure at different frequencies. The tangential velocity distribution has little influence. Resonance enhances the turbulence of the flow field and shifts the velocity axis.

3) At $33.36 \mathrm{~Hz}$, the vibration at different altitude positions is basically synchronous, presenting the first order mode; At $100 \mathrm{~Hz}$, the deformation of the structure is out of sync, and torsion occurs, and the second order mode is presented. At $200 \mathrm{~Hz}$, the structural deformation is small, the fluid-solid coupling effect is weak, and the third-order mode is presented.

\section{References}

1. PEI Ji, YUAN Shouqi, YUAN Jianping. Numerical calculation of fluid-structure coupling affect on internal flow field in a centrifugal pump[J]. Journal of agricultural machinery, 2009, 12: 107-112.

2. PEI Ji. The research of fluid-structure interaction mechanism of transient hydraulic vibration and the unsteady flow intensity of centrifugal pump[D]. Jiangsu university, 2013.

3. XU Feng. The fluid-solid coupling of simulation analysis and experimental research of piezoelectric pump[D]. Jilin university, 2015.

4. Satish Kumar Chimakurthi, Steve Reuss, Michael Tooley. ANSYS Workbench System Coupling: a state-of-the-art computational framework for analyzing multiphysics problems[J]. engineering with Computers. (2018) 34: 385-411.

5. Zhu Li, Yang Changming, Zheng Jun. Structural analysis of impellers of axial flow pumps based on fluid-solid interaction [J]. Fluid machinery. 2013, 41(3): 20-24.

6. Li Wei, Yang Yongfei, Shi Weidong. Study on mechanical characteristics of impeller of mixed-flow pump based on bidirectional fluid-solid interaction [J].Journal of Agricultural Machinery. 2015, 46(12): $82-88$

7. Liang Quanwei, Wang Zhengwei, Fangyuan. Modal
Analysis of Francis Turbine Runner Considering Fluid-Solid Coupling[J]. Journal of Hydropower. 2004, 23(3): 116-120.

8. Onur Celikkan, Haluk Erol. Vibroacoustic Analysis of a Refrigerator Freezer Cabinet Coupled with an Air Duct[J]. Advances in Acoustics and Vibration Volume. 2017, 1-18

9. QIU Yadong, WANG Zunce, LI Cuiyan, et al. The vibration characteristic research of variable diameter pipe of hydrocyclone under the fluid-structure coupling effect[J]. Chemical machinery, 2015, 02: 240-244.

10. LI Sen, ZHANG Jian, WANG Zunce, et al. The numerical simulation of flow field in the hydrocyclone under the action of fluid-structure coupling. Chemical machinery, 2015, 05: 706-710.

11. Xu Yan, Zhang Yanyue, Xu Dekui. Fluid-solid coupling study of spiral flow in cyclone separator under vibration condition[J]. Journal of China Petroleum University. 2017, 41(4): 140-147.

12. Yan Xu, Yan-yue Zhang, F.C.G.A Nicolleau, Zunce Wang, PIV of Swirling Flow in a Conical Pipe with Vibrating Wall[J]. International Journal Apply Mechanics, 2018, 10(2): 1850022. 